\title{
Spine Cancer PTX TNM Finding v8
}

National Cancer Institute

\section{Source}

National Cancer Institute. Spine Cancer PTX TNM Finding v8. NCI Thesaurus. Code C136583.

Spine cancer in which the primary tumor cannot be assessed. (from AJCC 8th Ed.) 